\title{
Use of immuno-oncology in melanoma
}

\author{
M.G. Smylie MBChBP*
}

\begin{abstract}
Treatment options for patients with metastatic melanoma have expanded rapidly since the approval of ipilimumab by the U.S. Food and Drug Administration in 2011. Cytokines such as interferon and interleukin-2 were approved in 1995 and 1998 respectively. However, the effect on survival was marginal, and the toxicity, substantial. Multiple vaccine studies likewise failed to show improvements in survival. The "Holy Grail" came with the discovery of immune checkpoints, and the first metastatic melanoma trial to show an improvement in overall survival involved the use of an immune checkpoint inhibitor against CTLA-4: ipilimumab. Since then, the field of immuno-oncology has exploded, with approvals for PD-1 inhibitors and discovery, in clinical trials, of several novel checkpoints such as TIM-3, LAG-3, and others. In fact more than 950 novel immunotherapy drugs are currently being trialled. Recently, combinations of CTLA- 4 and PD-1 inhibitors have been associated with 1-year survival rates exceeding $80 \%$ and 4 -year survival rates greater than $50 \%$. In no tumour has as much progress been made in the last 5 years as in melanoma, and the efforts to unravel and exploit mechanisms used by the tumour to avoid immune detection are just beginning.
\end{abstract}

Key Words Melanoma, immuno-oncology

Curr Oncol. 2020 April:27(S2)51-58

www.current-oncology.com

\section{REVIEW}

\section{Epidemiology}

The worldwide incidence of melanoma continues to increase dramatically. In the United States, melanoma is the 5th most common cancer in men and the 6th most common in women ${ }^{1}$. In Canada, estimates suggest that, in 2017, approximately 7200 new cases of melanoma were diagnosed, and approximately 1250 people died from the disease $^{2}$. In Canada, melanoma is the 6th most common cancer in men and the 7 th in women. The incidence rate for melanoma doubled in the United States from 1982 to 2011, and despite increased screening efforts and public education, mortality rates have remained constant.

Fortunately, most patients in Western countries present with thin lesions and have an excellent prognosis. Still, a small proportion will present with higher-risk thick lesions, and a yet smaller number present with metastatic disease. About $30 \%$ of patients with metastatic disease will come from the so-called good prognosis group.

\section{Historical Perspectives}

Historically, and until 2010, the treatment of metastatic melanoma was largely an exercise in futility. Based on phase II studies showing response rates of about $15 \%-20 \%{ }^{3}$, dacarbazine (DTIC) chemotherapy was approved by the U.S. Food and Drug Administration (FDA) in the 1970s and shortly thereafter by Health Canada. In the 1980s, based on higher response rates seen in phase II studies ${ }^{4}$, combination regimens such as cisplatin-vinblastine-DTIC and the 4-drug Dartmouth protocol (cisplatin-carmustineDTIC-tamoxifen) became the standard of care at many cancer centres. Subsequent randomized trials of cisplatinvinblastine-DTIC or the Dartmouth regimen compared with DTIC alone failed to show any survival advantage for the combination chemotherapy regimens-with more toxicity being observed with combination therapy ${ }^{5}$. At the time of the largest trial in which DTIC chemotherapy was used as the control arm (with oblimersen sodium being used in the intervention arm), single-agent DTIC was associated with a response rate of $7.5 \%{ }^{6}$.

Interleukin 2 was approved by Health Canada in 1998 based on durability of response, although the response rate was $16 \%$, and the complete response rate, $6 \%{ }^{7}$. Given the complexity of interleukin 2 treatment and its significant toxicity, only a few centres in Canada used it.

The U.S. National Comprehensive Cancer Network guideline for the management of metastatic melanoma said to enter affected patients onto clinical trials. And for 40 years, no agent was proved to be superior in survival to single-agent DTIC.

During the last 500 million years, the immune system has evolved to attack and kill anything that does not belong in our bodies, and yet it fails to reject many cancers. The natural selection and immuno-editing of cancer cells ensures that tumours that do develop either are immuneprivileged or are masters of manipulating and switching off the immune system. Use of the immune system to attack 
cancer is not a new phenomenon: In 1898, after reading a case report of regression in a sarcoma patient after an erysipelas infection, William Coley began to inject live Streptococcus pyogenes into cancer patients. That treatment was later modified to use heat-killed Streptococcus pyogenes and subsequently to add Serratia marcescens. Known as Coley's toxin, it was marketed by Parke Davis up until 1962. Multiple cytokines, vaccines, and immune adjuvants were subsequently tested in human trials, but none were associated with improved survival in randomized trials.

It was not until the identification of immune checkpoints on $\mathrm{T}$ cells that progress using the immune system to fight cancer resumed. First identified by Pierre Golstein and colleagues in $1987^{8}$, CTLA- 4 was subsequently shown by Tak Mak and colleagues in 1995 to be a negative regulator of $\mathrm{T}$ cell function ${ }^{9}$. Those negative regulators of $\mathrm{T}$ cell function then became known as immune checkpoints. In the 1990s, James Allison, with colleague Matthew Krummel, demonstrated that, by blocking CTLA- 4 with a monoclonal antibody, some cancers in mice could be cured ${ }^{10}$. Independently, in 1992, Tasuku Honjo and colleagues discovered a second immune checkpoint that was named programmed death receptor 1 (PD-1) ${ }^{11}$. The discovery by Allison and Krummel led to the development of ipilimum$\mathrm{ab}$, and Honjo's discovery of PD-1 led to the development of nivolumab and pembrolizumab. In 2018, Allison and Honjo received the Nobel Prize in Medicine or Physiology for their discoveries, which have led to an explosion in immune checkpoint therapy, known as immuno-oncology. Immuno-oncology has transformed the treatment of melanoma and multiple other cancers, and is now recognized as the so-called 4th pillar of cancer treatment, together with surgery, radiation, and chemotherapy ("slash, burn, and poison"). Although the science of immune checkpoints is not fully understood, that lack of understanding does not matter: the drugs are working, and the science will be revealed as advances continue.

\section{Immune Checkpoints}

Ipilimumab was tested in several phase I and II studies, and consistently, a small fraction of patients with stage IV melanoma were experiencing long-term remissions ${ }^{12}$. It was also noted that responses could be conventional or nonconventional, with some patients showing pseudoprogression before achieving a response. In addition, a unique side-effect profile, subsequently designated as immunerelated adverse events, was seen (Table I). The first randomized phase III study in pretreated patients was MDX010-020, which was presented at the American Society of Clinical Oncology 2010 annual meeting and published in the New England Journal of Medicine ${ }^{13}$. It randomized 600 patients in a 3:1:1 fashion to ipilimumab plus gp100 vaccine, to ipilimumab alone, or to gpl00 vaccine alone. The study was unique in that it used a dose of $3 \mathrm{mg} / \mathrm{kg}$ and only 4 treatments, when the more conventional dose at the time was $10 \mathrm{mg} / \mathrm{kg}$ and 4 treatments, with a maintenance phase of $10 \mathrm{mg} / \mathrm{kg}$ every 3 months till either disease progression or unacceptable toxicity. The study was powered to show an improvement in overall survival (os) for the ipilimumab-containing arms compared with gp100 vaccine, and not for ipilimumab plus gp100 vaccine compared with ipilimumab alone. The trial met its primary endpoint of a demonstrated improvement in os in metastatic melanoma. In 2011, the FDA subsequently approved the use of ipilimumab for any line of treatment in metastatic melanoma. The European Medicines Agency-and subsequently Health Canada-approved ipilimumab only in the second-line setting.

Because the FDA was privy to the results of MDX010024, which compared first-line ipilimumab-DTIC with DTIC-placebo, their approval was conditional on BristolMyers Squibb evaluating ipilimumab $10 \mathrm{mg} / \mathrm{kg}$ compared with $3 \mathrm{mg} / \mathrm{kg}$ to determine the more effective dose. The MDX010-024 study was likewise positive for survival, showing that, compared with chemotherapy alone, the addition of ipilimumab to DTIC chemotherapy was superior for survival ${ }^{14}$, albeit with increased toxicity and a $20 \%$ incidence of hepatotoxicity in the ipilimumab arm. A subsequent meta-analysis of more than 1800 patients treated in phase II and III studies of ipilimumab showed that the survival curve plateaued at 3 years with $22 \%$ survival, and the 5 -year survival rate was $20 \%$ (Figure 1$)^{15}$. Follow-up to 10 years has shown an ongoing survival plateau, and the

TABLE I Adverse events

\begin{tabular}{|c|c|c|c|}
\hline Event or management & $\begin{array}{l}\text { Ipilimumab } \\
\text { (311 patients) }\end{array}$ & $\begin{array}{l}\text { Nivolumab } \\
\text { (313 patients) }\end{array}$ & $\begin{array}{l}\text { Nivolumab-ipilimumab } \\
\text { (313 patients) }\end{array}$ \\
\hline \multicolumn{4}{|l|}{ Grade 3 or $4[n(\%)]$} \\
\hline Any & $173(55.6)$ & $136(43.5)$ & $215(68.7)$ \\
\hline Treatment-related & $85(27.3)$ & $51(16.3)$ & $172(55)$ \\
\hline Diarrhea & $19(6.1)$ & $7(2.2)$ & $29(9.3)$ \\
\hline Fatigue & $3(1.0)$ & $4(1.3)$ & $13(4.2)$ \\
\hline Pruritus & $1(0.3)$ & 0 & $6(1.9)$ \\
\hline Colitis & $27(8.7)$ & $2(0.6)$ & $24(7.7)$ \\
\hline Hepatic & $5(1.6)$ & $8(2.6)$ & $59(18.8)$ \\
\hline Treatment-related leading to discontinuation & $41(13.2)$ & $16(5.1)$ & $92(29.4)$ \\
\hline \multicolumn{4}{|l|}{ Management of events (\%) } \\
\hline With immune modulatory agents, including topical agents & 55.9 & 47 & 83.4 \\
\hline With secondary immunosuppressive agents ${ }^{\mathrm{a}}$ & 5.1 & 0.6 & 6.1 \\
\hline
\end{tabular}

a For example, infliximab. 


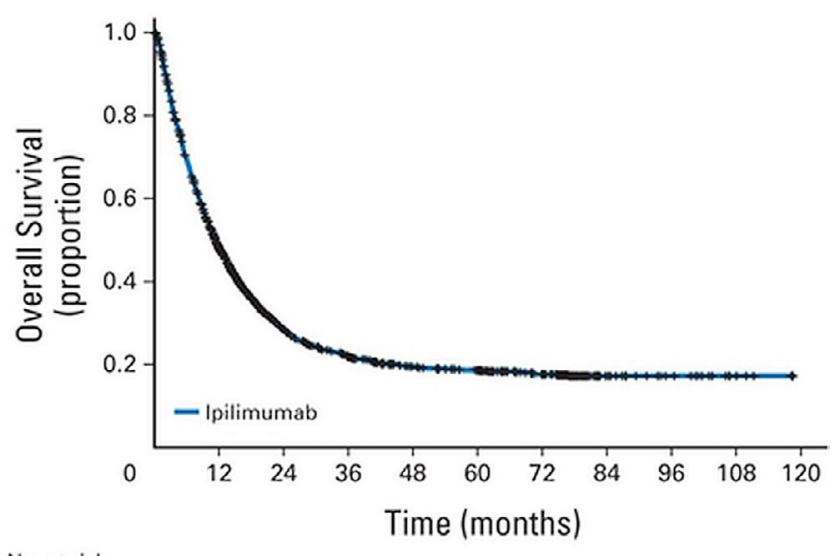

No. at risk

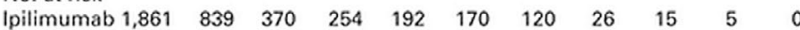

FIGURE 1 Primary analysis of pooled overall survival (OS) data. Individual patient data were pooled from ten prospective trials and two retrospective observational studies of ipilimumab in metastatic melanoma ( $n=1861)$. Median OS was 11.4 months [95\% confidence interval $(\mathrm{Cl})$ : 10.7 months to 12.1 months] with a 3 -year survival rate of $22 \%$ (95\% Cl: $20 \%$ to $24 \%)$.

FDA now allows ipilimumab to be marketed as a potentially curative treatment in metastatic melanoma.

Another CTLA-4 antibody, tremelimumab, was also tested in a randomized trial comparing it with DTIC or temozolomide. Tremelimumab was given at $15 \mathrm{mg} / \mathrm{kg}$ every 90 days. The study was stopped early when it became clear that the primary os endpoint would not be met ${ }^{16}$. The every90-days dose was chosen based on an earlier randomized phase II study that compared $10 \mathrm{mg} / \mathrm{kg}$ every 4 weeks with $15 \mathrm{mg} / \mathrm{kg}$ every 90 days. There was no difference in survival between the two arms, but less toxicity was associated with the every-90-days regimen ${ }^{17}$. The median number of doses in the tremelimumab arm was 1.1, and a retrospective assessment showed that a significant number of patients in the chemotherapy arm received ipilimumab through either a compassionate access program or a clinical trial, which could therefore have affected the survival rate.

The PD-1 checkpoint was first described by Honjo and colleagues in 1995. Nivolumab is a fully human immunoglobulin G4 monoclonal inhibitor of PD-1, and pembrolizumab is a humanized immunoglobulin G4-k anti-PD-1 antibody.

In 2011, Topalian et al. ${ }^{18}$ reported the phase I results for a PD-1 inhibitor, nivolumab, in 5 different tumour sites. The objective response rate (ORR) in pretreated patients with melanoma was $28 \%$. Likewise, Hamid et al. ${ }^{19}$ reported a $38 \%$ response rate to pembrolizumab in pretreated melanoma patients. The CheckMate 037 study compared nivolumab with standard-of-care chemotherapy in ipilimumabpretreated patients. The co-primary endpoints were an improvement in ORR and os. Compared with chemotherapy alone, nivolumab was associated with an improved ORR ( $27 \%$ vs. $13 \%$ ) and median duration of response (32 months vs. 13 months), with fewer grade 3 or 4 adverse events. Although the trial showed an improvement in the oRR, it failed meet its other co-primary endpoint: improvement in $\mathrm{OS}^{20}$. An imbalance of patients with brain metastases and higher serum lactate dehydrogenase in the nivolumab arm might have confounded the survival differences.

In the BMS 066 study, patients with untreated $B R A F$ wild-type metastatic melanoma were randomized 1:1 to either DTIC or nivolumab $(n=418)^{21}$. The study was blinded, with no plans for crossover. The 1-year os rate was $72.9 \%$ in the nivolumab arm [95\% confidence interval (CI): $65.5 \%$ to $78.9 \%$ ] and $42.1 \%$ in the DTIC arm (95\% CI: 33.0 to 50.9$)$. The ORR was $40 \%$ with nivolumab (95\% CI: $33.3 \%$ to $47.0 \%$ ) and $13.9 \%$ with DTIC (95\% CI: $9.5 \%$ to $19.4 \%$ ). On 10 June 2014 , the data and safety monitoring committee recommended early closure of the trial because an unplanned analysis showed an improvement in os in favour of the nivolumab arm. In that study, DTIC had been chosen as the comparator because, at the time, ipilimumab was not approved in the first-line setting in most regions outside of the United States. The results of the study led to the approval by the FDA-and subsequently by Health Canada-of nivolumab in the first-line setting for patients with unresectable metastatic $B R A F$ wild-type melanoma.

In the KEYNOTE-006 first-line study of pembrolizumab compared with ipilimumab, 834 patients were randomized 1:1:1 to receive pembrolizumab $10 \mathrm{mg} / \mathrm{kg}$ every 2 weeks, or pembrolizumab $10 \mathrm{mg} / \mathrm{kg}$ every 3 weeks, or ipilimumab $3 \mathrm{mg} / \mathrm{kg}$ every 3 weeks for 4 doses $^{22}$. The co-primary endpoints were progression-free survival (PFS) and os. The 6 -month PFS rates were $47.3 \%$ for pembrolizumab every 2 weeks, $46.4 \%$ for pembrolizumab every 3 weeks, and $26.5 \%$ for ipilimumab [hazard ratio (HR) for disease progression: $0.58 ; p<0.001$ for both pembrolizumab regimens compared with ipilimumab; 95\% confidence intervals (CIs): 0.46 to 0.72 and 0.47 to 0.72 respectively]. The study was not powered to detect a difference between the two pembrolizumab arms. Estimated 12-month survival rates were $74.1 \%, 68.4 \%$, and $58.2 \%$ respectively (HR for death, pembrolizumab every 2 weeks: 0.63 ; $95 \%$ CI: 0.47 to $0.83 ; p=0.0005$; HR for death, pembrolizumab every 3 weeks: 0.69 ; $95 \%$ CI: 0.52 to 0.90 ; $p=0.0036$ ). Response rates were $33.7 \%$ for pembrolizumab every 2 weeks, $32.9 \%$ for pembrolizumab every 3 weeks, and $11.9 \%$ for ipilimumab. The toxicity profiles favoured the pembrolizumab arms. The final os was updated by Schachter et $a l .{ }^{23}$ in 2017. Median os had not been reached in the pembrolizumab arms: 22.1 months to not reached in the 2-week group, and 23.5 to not reached in the 3-week group, compared with 16 months in the ipilimumab arm (range: 13.5-22 months).

The FDA - and subsequently Health Canada-approved pembrolizumab $2 \mathrm{mg} / \mathrm{kg}$ given every 3 weeks in the first or second line for melanoma. The $2 \mathrm{mg}$ dose was accepted because the phase i study involving more than 600 patients did not seem to show a difference between $2 \mathrm{mg}$ and $10 \mathrm{mg}$, except for more toxicity in the $10 \mathrm{mg}$ arm.

Compared with ipilimumab or chemotherapy, PD-1 inhibitors have consistently been associated with improvements in response rates, PFS, and 1- and 2-year survival rates (Table II).

\section{Combination Checkpoint Therapy}

Because CTLA- 4 blockade works at the priming phase in secondary lymphoid tissues, and PD-1 blockade works at the effector phase, a phase I study set out to assess various 
doses of nivolumab and ipilimumab given in combination $^{24}$. The encouraging 1- and 2-year survival rates led to a phase III study, BMS 067, in which 945 patients were allocated 1:1:1 to receive ipilimumab $3 \mathrm{mg} / \mathrm{kg}$ plus nivolumab $1 \mathrm{mg} /$ kg every 3 weeks for 4 doses and then nivolumab maintenance $3 \mathrm{mg} / \mathrm{kg}$ every 3 weeks, or nivolumab $3 \mathrm{mg} / \mathrm{kg}$ every 3 weeks, or ipilimumab $3 \mathrm{mg} / \mathrm{kg}$ every 3 weeks for 4 doses ${ }^{25}$. The co-primary endpoints were PFS and os. The study was powered to compare the nivolumab-containing arms with the ipilimumab arm, and not ipilimumab-nivolumab with nivolumab alone. The median PFS was 11.5 months with ipilimumab-nivolumab (95\% CI: 8.9 to 16.7 months) compared with 2.9 months with ipilimumab (95\% CI: 2.8 to 3.4 months; HR for death or disease progression: $0.42 ; 99.5 \%$ CI: 0.31 to $0.57 ; p<0.001$ ) and 6.9 months with nivolumab alone (95\% CI: 4.3 to 9.5 months; HR for the comparison with ipilimumab: 0.57 ; $99.5 \%$ CI: 0.43 to $0.76: p=0.001$ ). Grade 3 or 4 treatment-related adverse events occurred in $55 \%$ of the patients receiving ipilimumab-nivolumab (Table I), in
$16.3 \%$ of those receiving nivolumab alone, and in $27.3 \%$ of those receiving ipilimumab alone. A recent update showed 4 -year survival rates of $53 \%, 46 \%$, and $30 \%$ for ipilimumabnivolumab, nivolumab, and ipilimumab respectively. The phase I trial of ipilimumab-nivolumab had shown less toxicity when a lower dose of ipilimumab $(1 \mathrm{mg} / \mathrm{kg})$ and standard-dose nivolumab ( $3 \mathrm{mg} / \mathrm{kg}$ ) were used (Table III).

The CheckMate 511 study randomized 360 patients with untreated metastatic melanoma to either ipilimumab $3 \mathrm{mg} / \mathrm{kg}$ plus nivolumab $1 \mathrm{mg} / \mathrm{kg}$ or to ipilimumab $1 \mathrm{mg} / \mathrm{kg}$ plus nivolumab $3 \mathrm{mg} / \mathrm{kg}^{26}$. The primary endpoint was the incidence of grades 3-5 adverse events. The study met its primary endpoint, showing a grade 3 or 4 adverse event rate of $33.9 \%$ in the ipilimumab $1 \mathrm{mg}$ arm compared with $48.3 \%$ in the ipilimumab $3 \mathrm{mg}$ arm. The study was not powered to look at OS, but PFS was 9.9 months with ipilimumab $3 \mathrm{mg} / \mathrm{kg}$ and $8.9 \mathrm{months}$ with ipilimumab $1 \mathrm{mg} / \mathrm{kg}$, and 12 -month os was $81 \%$ with nivolumab $1 \mathrm{mg} / \mathrm{kg}$ plus ipilimumab $3 \mathrm{mg} / \mathrm{kg}$ and $79.7 \%$ with nivolumab $3 \mathrm{mg} / \mathrm{kg}$

TABLE II Trials of PD-1 inhibitors

\begin{tabular}{|c|c|c|c|c|}
\hline \multirow[t]{2}{*}{ Trial variable } & \multicolumn{4}{|c|}{ Trial name } \\
\hline & CheckMate 067 & CheckMate 066 & KEYNOTE-006 & CheckMate 037 \\
\hline Investigational drug & Nivolumab & Nivolumab & $\begin{array}{l}\text { Pembrolizumab every } 2 \text { weeks; } \\
\text { pembrolizumab every } 3 \text { weeks }\end{array}$ & Nivolumab \\
\hline Comparator & Ipilimumab & Dacarbazine & Ipilimumab & $\begin{array}{l}\text { Investigator's choice } \\
\text { chemotherapy }\end{array}$ \\
\hline ORR (\%) & 43.7 & 40 & 33.7 vs. 32.9 & 27 \\
\hline Median PFS (months) & 6.9 & 5.1 & 5.5 vs. 4.1 & 3.1 \\
\hline \multicolumn{5}{|l|}{ Survival (\%) } \\
\hline 1 Year & 74 & 73 & 74 vs. 68 & 58.9 \\
\hline 2 Year & 59 & 57.7 (estimated) & 55 vs. 55 & 38.7 \\
\hline Median OS (months) & NR & NR & NR vs. NR & 15.7 \\
\hline
\end{tabular}

ORR = objective response rate; $\mathrm{PFS}=$ progression-free survival; $\mathrm{OS}=$ overall survival; $\mathrm{NR}=$ not reported .

TABLE III Trials of PD-1 and CTLA-4 inhibitor combinations

\begin{tabular}{|c|c|c|c|}
\hline \multirow[t]{2}{*}{ Trial variable } & \multicolumn{3}{|c|}{ Trial name } \\
\hline & CheckMate 069 & KEYNOTE-029 & CheckMate 067 \\
\hline Investigational drug & Ipilimumab & $\begin{array}{l}\text { Pembrolizumab-ipilimumab } \\
\text { (phase IB) }\end{array}$ & Nivolumab \\
\hline Comparator & Ipilimumab-nivolumab & - & Ipilimumab-nivolumab \\
\hline ORR (\%) & 11 vs. 59 & 62 & 44.6 vs. 58.3 \\
\hline PFS (months) & 2.9 vs. 11.5 & NR & 6.9 vs. 11.5 \\
\hline OS (months) & NR & NR & 36.9 vs. NR \\
\hline \multicolumn{4}{|l|}{ Survival (\%) } \\
\hline 1 Year & & 88 & 74 vs. 73 \\
\hline 2 Year & 53.6 vs. 63.8 & 75 & 59 vs. 64 \\
\hline 3 Year & & 73 & 51 vs. 58 \\
\hline 4 Year & & NR & 46 vs. 53 \\
\hline 5 Year & NR & NR & NR \\
\hline
\end{tabular}

ORR = objective response rate; PFS = progression-free survival; NR = not reported; OS = overall survival. 
plus ipilimumab $1 \mathrm{mg} / \mathrm{kg}$. Response rates were $45.6 \%$ for ipilimumab $1 \mathrm{mg} / \mathrm{kg}$ plus nivolumab $3 \mathrm{mg} / \mathrm{kg}$ (95\% CI: $38.1 \%$ to $53.1 \%$ ) compared with $50.6 \%$ for ipilimumab $3 \mathrm{mg} / \mathrm{kg}$ and nivolumab $1 \mathrm{mg} / \mathrm{kg}$ (95\% CI: $43.0 \%$ to $58.1 \%$ ). In summary, there seemed to be no difference in efficacy parameters (although the study was not powered to look at the efficacy parameters), but rather a statistically significant difference in toxicity.

At 12 medical centres, KEYNOTE-029, an open-label phase IB study, enrolled 153 patients with unresectable stage III or IV melanoma who had received no prior immunotherapy ${ }^{27}$. Patients were treated with standard doses of pembrolizumab $(2 \mathrm{mg} / \mathrm{kg})$ and ipilimumab $(1 \mathrm{mg} / \mathrm{kg})$ every 3 weeks for 4 doses, and then pembrolizumab $2 \mathrm{mg} / \mathrm{kg}$ every 3 weeks for up to a maximum of 2 years or until disease progression or unacceptable toxicity. The primary endpoints were safety and toxicity, with secondary endpoints of ORR and os. Grades 3-4 treatment-related adverse events occurred in $45 \%$ of patients, with no grade 5 events. Estimated 1-year PFS was $69 \%$ (95\% CI: $60 \%$ to $75 \%$ ), and estimated 1-year survival was $89 \%$ (95\% CI: $83 \%$ to $93 \%$ ). The ORR was $61 \%(95 \%$ CI: $53 \%$ to $69 \%$ ). An update at the Society for Melanoma Research 2018 meeting reported an ORR of $62 \%$, including a $27 \%$ complete response rate, a 3 -year PFS of $59 \%$, and an os of $73 \%$. At 3 years, $83 \%$ of responses were ongoing.

A randomized phase II study of alternative dosing strategies of pembrolizumab-ipilimumab is ongoing. Because most of the toxicity associated with combination regimens appears to be related to the higher dose of ipilimumab, the lower dose used in the two earlier studies would make the regimen more palatable for oncologists. Randomized studies to show how to properly sequence the two immune checkpoint inhibitors or whether they are better used concurrently are still lacking. Real-world data will hopefully help in deciding how to use these active regimens. In Canada, access has been limited to CTLA- 4 inhibitor after progression on a PD-1 inhibitor, and thus access is limited when the agents are used sequentially; more combination regimens might therefore be used.

At our centre, the preference for treatment is to use immunotherapy combination regimens in the first line for patients with a good Eastern Cooperative Oncology Group performance status and no relative contraindications to immunotherapy such as underlying autoimmune disease or other medical morbidities. Our decision is based on ongoing follow-up from the CheckMate 067 study, which showed improvements in survival extending to beyond 4 years, and a significantly larger number of patients remaining free of subsequent treatment. A retrospective review of patients with metastatic melanoma treated with immunotherapy in Alberta since 2008 identified 380 patients, of whom 80 received combination ipilimumabnivolumab as first-line therapy, and 139 received either first-line nivolumab or pembrolizumab without subsequently receiving ipilimumab. The 1- and 2-year survivals were, respectively, $87.2 \%$ and $75.9 \%$ for the combination and $56.1 \%$ and $42.6 \%$ for the PD- 1 therapies. Many patients treated with the combination regimen received their treatment at a time when ipilimumab had been defunded by all provinces except Quebec and was therefore not available to patients in Alberta.
New immunotherapy developments are outpacing the understanding of the science. Novel approaches such as manipulation of the tumour microenvironment, use of oncolytic viruses, and combinations with chemotherapy, radiation therapy, and other immune checkpoint inhibitors or agonist antibodies are now in clinical trials. Currently, more than 940 "new" immunotherapeutic drugs are being investigated, involving more than half a million patients worldwide. The key question in the future will be whether a T cell-deficient tumour can be converted into a T cell-high tumour.

Preclinical studies looking at ablation of chemokines such as CXCL1 reported inhibition of myeloid cell infiltration into the tumour and increased movement of dendritic cells, followed by CD8 cells, into the tumour ${ }^{28}$. The phase III randomized study of pembrolizumab with or without the IDO inhibitor epacadostat failed to show any improvement in PFS or $\mathrm{OS}^{29}$. Likewise, re-education of macrophages from the M2 phenotype to the M1 phenotype by blockade of CSF-1 receptors is possible. Other immune checkpoints such as LAG-3, TIM-23, GITR are in phase I/II trials to assess their efficacy as either single agents or in combination with anti-PD-1 therapy. Chemotherapy drugs such as gemcitabine and 5-fluorouracil are toxic to myeloid-derived suppressor cells, which are critical in maintaining a hostile tumour microenvironment. The future is bright, and the next 5 years will no doubt lead to ever-expanding treatment algorithms.

\section{Treatment of Brain Metastases}

Brain metastases have historically carried an ominous prognosis, with most patients surviving only a few months. Immune checkpoint inhibitors are associated with encouraging response rates in the brain, with response durability equivalent to that seen in responses outside the brain.

A small study of 18 patients with brain metastases from melanoma showed a response rate of $22 \%^{30}$. Tawbi et $a{ }^{31}{ }^{31}$ recently reported the results of a phase II trial that included 94 patients with metastatic melanoma and at least 1 measurable nonirradiated tumour in the brain. Patients had to be asymptomatic, with a tumour diameter of at least $5 \mathrm{~mm}$ and no greater than $3 \mathrm{~cm}$. The overall intracranial response rate was $55 \%$, with a complete response rate of $26 \%$. In the initial assessment, the estimated 6-, 9-, and 12 -month survivals were $92.35 \%, 82.8 \%$, and $81.5 \%$ respectively. Likewise, Long et al. ${ }^{32}$ published the results of a multicentre phase II study in Australia comparing combination ipilimumab-nivolumab with nivolumab alone in patients with central nervous system metastases from melanoma. Patients with untreated asymptomatic brain metastases were randomized to ipilimumab-nivolumab (cohort A) or to nivolumab alone (cohort B). Cohort $\mathrm{C}$ consisted of patients with brain metastases for whom local therapy had failed, or who were experiencing neurologic symptoms, or who had leptomeningeal disease; they were treated with nivolumab. Between November 2014 and April 2017, 79 patients were enrolled: 36 in cohort A, 27 in cohort B, and 16 in cohort $C$. The intracranial response rate was $46 \%$ in cohort A (95\% CI: $29 \%$ to $63 \%$ ), $20 \%$ in cohort B (95\% CI: $7 \%$ to $41 \%)$, and $6 \%$ in cohort C (95\% CI: $0 \%$ to $30 \%)$. Intracranial complete responses occurred in $17 \%$ of cohort A, $12 \%$ of cohort B, and $13 \%$ of cohort $\mathrm{C}$. 
At our institution, radiation for patients with small, asymptomatic brain metastases proceeding to targeted or immune therapy is no longer routinely recommended unless intracranial progression is evident.

\section{Adjuvant Therapy}

The use of targeted therapy and immune checkpoint therapy in the adjuvant setting has now become the standard of care for patients with resected stage IIIA (nodal metastases $>1 \mathrm{~mm}$ ), stages IIIB-D, and stage IV disease. The use of high-dose interferon was approved in 1995 for resected stages IIB and III disease based on the Eastern Cooperative Oncology Group 1684 study. However, the follow-up Eastern Cooperative Oncology Group 1697 study failed to confirm the survival benefit, and that result, together with a meta-analysis showing an improvement in relapse-free survival, but minimal benefit in os, and the associated toxicity led many oncologists to abandon interferon.

The first study of an immune checkpoint inhibitor in the adjuvant setting was the European Organisation for Research and Treatment of Cancer 18071 study reported by Eggermont et al. ${ }^{33}$. The os rate at 5 years was $65.4 \%$ in the ipilimumab group and $54.4 \%$ in the placebo group (HR for death: $0.72 ; 95.1 \%$ CI: 0.58 to $0.88 ; p=0.001)$. Ipilimumab was approved by the FDA at a dose of $10 \mathrm{mg} / \mathrm{kg}$ given every 3 weeks for induction, followed by maintenance doses of $10 \mathrm{mg} / \mathrm{kg}$ every 3 months for up to 3 years. That regimen was submitted to the European Medicines Agency, but the application was subsequently withdrawn because of ongoing questions about the dose. The approved dose in metastatic disease was $3 \mathrm{mg} / \mathrm{kg}$ given every 3 weeks for 4 doses, with no maintenance therapy.

Adjuvant studies with PD-1 inhibitors are showing improvements in PFS with both nivolumab and pembroli$z^{2}$ mab $^{34,35}$. The BMs 238 study compared nivolumab with ipilimumab as adjuvant therapy in patients with resected stages IIIB-C and IV disease. At a minimum follow-up of 18 months, the 12-month rate of recurrence-free survival was $70.5 \%$ in the nivolumab group (95\% CI: $66.1 \%$ to $74.5 \%$ ) and $60.8 \%$ in the ipilimumab group (95\% CI: $56.0 \%$ to $65.2 \%$; HR for disease recurrence or death: $0.65 ; 97.56 \%$ CI: 0.51 to $0.83 ; p<0.001)$. The Merck 054 study compared adjuvant pembrolizumab with placebo in resected stages IIIA (lymph node metastases $>1 \mathrm{~mm}$ ) and IIIB-C disease. At a median follow-up of 15 months, recurrence-free survival was significantly longer with pembrolizumab than with placebo in the overall intention-to-treat population [1-year rate of recurrence-free survival: $75.4 \%$ (95\% CI: $71.3 \%$ to $78.9 \%$ ) vs. $61.0 \%$ (95\% CI: $56.5 \%$ to $65.1 \%$ ); HR for recurrence or death: $0.57 ; 98.4 \%$ CI: 0.43 to $0.74 ; p<0.001$ ]. The study allowed crossover for patients randomized to the placebo arm, such that they could receive pembrolizumab at relapse. That approach will help to define whether os can be improved by giving the drug in the adjuvant setting or by treating only patients who relapse. Longer follow-up for os will be required in that population. Currently, no available evidence favours the choice of a particular PD-1 inhibitor, nor the choice of targeted therapy compared with immune checkpoint therapy, in patients positive for $B R A F$ mutation.

In locally advanced stage III disease, Rozeman et al. ${ }^{36}$ described results of a multicentre 3-arm study in which patients with resectable stage III disease, no in-transit metastases, and normal serum lactate dehydrogenase were randomized to ipilimumab $3 \mathrm{mg} / \mathrm{kg}$ plus nivolumab $1 \mathrm{mg} / \mathrm{kg}$ every 3 weeks for 2 cycles ( $\operatorname{arm~A}$ ), or ipilimumab $1 \mathrm{mg} / \mathrm{kg}$ plus nivolumab $3 \mathrm{mg} / \mathrm{kg}$ every 3 weeks for 2 cycles (arm B), or ipilimumab $3 \mathrm{mg} / \mathrm{kg}$ every 3 weeks, immediately followed by 2 cycles of nivolumab $3 \mathrm{mg} / \mathrm{kg}$ every 2 weeks (arm $\mathrm{C}$ ). The radiologic response rates were $60 \%, 60 \%$, and $42 \%$ respectively, and the pathologic response rates were $80 \%$ [ $43 \%$ pathologic complete response (pcR)], $77 \%$ (57\% pcR), and 68\% (24\% pcR) respectively. No patient with a pathologic response has relapsed, but 9 of 21 patients with no pathologic response relapsed. Of 2 patient deaths, 1 was attributed to melanoma ( $\operatorname{arm~A,~no~pathologic~}$ response), and 1, to complications after an immune-related encephalitis 9.5 months after the start of therapy (arm A, pCR). Arm $\mathrm{C}$ was dropped per the recommendation of the data and safety monitoring committee because of increased toxicity in that arm. Neoadjuvant ipilimumab $1 \mathrm{mg} / \mathrm{kg}$ plus nivolumab $3 \mathrm{mg} / \mathrm{kg}$ was associated with substantially lower toxicity and yet seemed to preserve the high response rates. Neoadjuvant therapy also allows for an assessment of the effectiveness of immunotherapy early, with therapy able to be abandoned in patients who show no response.

\section{SUMMARY}

As the understanding of the immune system and its subsequent manipulation to fight tumours evolves over time, treatment algorithms will change, hopefully continuing to improve survival for patients. Currently, no good biomarkers have been identified to help in the decision about who should receive single-agent PD-1 inhibitors compared with combination PD-1 and CTLA- 4 inhibitors. In melanoma, PD-1 percentage has not been helpful in guiding therapy, and melanoma patients in Canada are thus not routinely tested. A recent presentation by Weber et $a l .{ }^{37}$ at the American Society of Clinical Oncology annual meeting showed that elevated serum C-reactive protein and interleukin 6 predict a poorer outcome in patients treated with immunotherapy. A prospective trial is now underway to look at the combination of an interleukin 6 inhibitor (such as tocilizumab) and a PD-1 inhibitor in that population. Longer follow-up of the combination trials will yield a better idea about long-term survival. Trials are now ongoing to assess how best to sequence targeted therapy and immunotherapy, or to use the combination of targeted agents and PD-1 inhibitors to treat patients with $B R A F$ mutations. At our centre, our bias is to use PD- 1 and CTLA- 4 inhibitors in combination for patients with a good performance status and without medical or autoimmune conditions that would be a relative contraindication to immunotherapy.

Immune checkpoint therapy has revolutionized the treatment of metastatic melanoma, and long-term durable remissions are now possible with 5-year survival rates of $35 \%$ or greater. The nihilism of metastatic melanoma authenticated in papers such as the meta-analysis of phase II melanoma trials published by Korn et al. ${ }^{38}$ in 2008 showing a median survival of 6.7 months has quickly been supplanted by optimism after several randomized trials showing significant survival benefits. In fact, many of the 
long-term survivors reported in the immuno-oncology trials appear to be cured of their disease. We are just at the overture of the work in immuno-oncology, and in the next 5 years, we can hope to see better understanding, with improvements in outcomes and, ultimately, survival. More data from randomized trials and real-world experience are being gathered to better define how to best make use of immune checkpoint inhibitors-sequentially or, if the added increase in toxicity can be justified, in combination.

\section{Future Directions}

The future of immunotherapy is bright-from drugs that can manipulate the tumour microenvironment in an attempt to facilitate immune-cell infiltration, to oncolytic viruses, combinations of immune agents with chemotherapy and radiation, and novel immune checkpoint inhibitors or agonist antibodies currently in clinical trials. "Re-education" of macrophages from the M2 phenotype to the M1 phenotype by blockade of CSF-1 receptors is possible. The CSF-1 receptor inhibitor studies are currently in phase I, as are studies of inhibitors of adenosine metabolism, which is profoundly immunosuppressive in the tumour microenvironment. Chemotherapy drugs such as gemcitabine and 5-fluorouracil are toxic to myeloid-derived suppressor cells, which are critical in maintaining a hostile tumour microenvironment.

Other immune checkpoints such as LAG-3, GITR, and TIM-3 are also being investigated as potential targets. A recent study by Ascierto et al. ${ }^{39}$ looked at the combination of a novel inhibitor of LAG-3, BMS-986016, in combination with nivolumab in patients who experienced progressive disease after receiving prior anti-PD-1/-L1 with or without anti-CTLA- 4 or BRAF or MEK inhibitors. The ORR was $16 \%$, and the disease control rate was $45 \%$.

The development of new drugs is exceeding the understanding of the science, and how best to sequence those new drugs or add them into existing combinations is an ongoing problem. Proof-of-concept is needed and is typically assessed in an immuno-refractory population. It has been more than 120 years since Coley first tried to manipulate the immune system, but at last, the promise of immunotherapy is coming to fruition.

\section{CONFLICT OF INTEREST DISCLOSURES}

I have read and understood Current Oncology's policy on disclosing conflicts of interest, and I declare the following interests: I have received honoraria for advisory boards and speaking engagements from Novartis, Bristol-Myers Squibb, Roche, and Merck.

\section{AUTHOR AFFILIATIONS}

*Cross Cancer Institute, Edmonton, AB.

\section{REFERENCES}

1. Siegel RL, Miller KD, Jemal A. Cancer statistics, 2017. $C A$ Cancer J Clin 2017;67:7-30.

2. Canadian Cancer Society's Advisory Committee on Cancer Statistics. Canadian Cancer Statistics 2017. Toronto, ON: Canadian Cancer Society; 2017.

3. Anderson CM, Buzaid AC, Legha SS. Systemic treatments for advanced cutaneous melanoma. Oncology (Williston Park) 1995;9:1149-58.

4. Del Prete SA, Maurer LH, O’Donnell J, Forcier RJ, LeMarbre P. A combination chemotherapy with cisplatin, carmustine, dacarbazine, and tamoxifen in metastatic melanoma. Cancer Treat Rep 1984;68:1403-5.

5. Chapman PB, Einhorn LH, Meyers ML, et al. Phase III multicenter randomized trial of the Dartmouth regimen versus dacarbazine in patients with metastatic melanoma. J Clin Oncol 1999;17:2745-51.

6. Bedikian AY, Millward M, Pehamberger H, et al. Bcl-2 antisense (oblimersen sodium) plus dacarbazine in patients with advanced melanoma: the Oblimersen Melanoma Study Group. J Clin Oncol 2006;24;4738-45.

7. Atkins MB, Lotze MT, Dutcher JP, et al. High-dose recombinant interleukin-2 therapy for patients with metastatic melanoma: analysis of 270 patients treated between 1985 and 1993. J Clin Oncol 1999;17:2105-16.

8. Brunet JF, Denizot F, Luciani F, et al. A new member of the immunoglobulin superfamily—CTLA-4. Nature 1987;328:267-70.

9. Waterhouse P, Penninger JM, Timms E, et al. Lymphoproliferative disorders with early lethality in mice deficient in CTLA-4. Science 1995;270:985-8.

10. Krummel MF, Allison JP. CD28 and CTLA-4 have opposing effects on the response of T cells to stimulation. J Exp Med 1995;182:459-65.

11. Ishida Y, Agata Y, Shibahara K, Honjo T. Induced expression of PD-1, a novel member of the immunoglobulin gene superfamily, upon programmed cell death. EMBO J 1992;11:3887-95.

12. Wolchuk J, Neyns B, Linette G, et al. Ipilimumab monotherapy in patients with advanced melanoma: a randomized, doubleblind, multicenter, phase II dose-ranging study. Lancet Oncol 2010;11:155-64.

13. Hodi FS, O’Day SJ, McDermott DF, et al. Improved survival with ipilimumab in patients with metastatic melanoma. NEngl J Med 2010;363:711-23.

14. Robert C, Thomas L, Bondarenko I, et al. Ipilimumab plus dacarbazine for previously untreated metastatic melanoma. N Engl J Med 2011;364:2517-26.

15. Schadendorf D, Hodi FS, Robert C, et al. Pooled analysis of long-term survival data from phase II and phase III trials of ipilimumab in unresectable or metastatic melanoma. JClin Oncol 2015;33:1889-94.

16. Ribas A, Kefford R, Marshall MA, et al. Phase III clinical trial comparing tremelimumab with standard of care chemotherapy in patients with advanced melanoma. J Clin Oncol 2013;31:616-22.

17. Camacho LH, Antonia S, Sosman J, et al. Phase I/II trial of tremelimumab in patients with metastatic melanoma.JClin Oncol 2009;27:1075-81.

18. Topalian SL, Hodi FS, Brahmer JR, et al. Safety, activity and immune correlates of anti-PD-1 antibody in cancer. $N$ Engl J Med 2012;366:2443-54.

19. Hamid O, Robert C, Daud A, et al. Safety and tumor responses with lambrolizumab (anti-PD-1) in melanoma. NEnglJ Med 2013;369:134-44. [Erratum in: N Engl J Med 2018;379:2185]

20. Larkin J, Minor D, D'Angelo S, et al. Overall survival in patients with advanced melanoma who received nivolumab versus investigator's choice chemotherapy in CheckMate 037: a randomized, controlled, open-label phase III trial. J Clin Oncol 2018;36:383-90.

21. Robert C, Long G, Brady B, et al. Nivolumab in previously untreated melanoma without BRAF mutation. $N$ Engl J Med 2015;372:320-30.

22. Robert C, Schachter J, Long GV. Pembrolizumab versus ipilimumab in advanced melanoma. $N$ Engl J Med 2015;372: 2521-32.

23. Schachter J, Ribas A, Long GV, et al. Pembrolizumab versus ipilimumab for advanced melanoma: final overall survival results of a multicentre, randomised, open-label phase 3 study (KEYNOTE-006). Lancet 2017;390:1853-62. 
24. Wolchok JD, Kluger H, Callahan MK, et al. Nivolumab plus ipilimumab in advanced melanoma. $N$ Engl J Med 2013;369:122-33. [Erratum in: N Engl J Med 2018;379:2185]

25. Larkin J, Chiarion-Sileni V, Gonzalez R, et al. Combined nivolumab and ipilimumab in untreated melanoma. NEngl $J$ Med2015;373:23-34. [Erratum in: NEnglJMed 2018;379:2185]

26. Lebbe C, Meyer N, Mortier L, et al. Evaluation of two dosing regimens for nivolumab in combination with ipilimumab in patients with advanced melanoma: the results from the phase III/IV CheckMate 511 trial. JClin Oncol 2019;37:867-75.

27. Long GV, Atkinson V, Cebon JS, et al. Standard-dose pembrolizumab in combination with reduced-dose ipilimumab for patients with advanced melanoma (KEYNOTE-029): an open-label, phase 1b trial. Lancet Oncol 2017;18:1202-10.

28. Li J, Byrne KT, Yan F, et al. Tumor cell-intrinsic factors underlie heterogeneity of immune cell infiltration and response to immunotherapy. Immunity 2018;49:178-93.

29. Long GV, Dummer R, Hamid O, etal. Epacadostat plus pembrolizumab versus placebo plus pembrolizumab in patients with unresectable or metastatic melanoma (ECHO-301/KEYNOTE-252): a phase 3, randomised, double-blind study. Lancet Oncol 2019; 20:1083-97.

30. Goldberg SB, Gettinger SN, Mahajan A, et al. Pembrolizumab for patients with melanoma or non-small-cell lung cancer and untreated brain metastases. Early analysis of a non-randomized, open label phase 2 study. Lancet Oncol 2016;17:976-83.

31. Tawbi HA, Forsyth PA, Algazi A, et al. Combined nivolumab and ipilimumab in melanoma metastatic to the brain. NEngl J Med 2018;379;722-30.

32. Long GV, Atkinson V, Lo S, et al. Combination nivolumab and ipilimumab or nivolumab alone in melanoma brain metastases: a multicenter randomized phase 2 study. Lancet Oncol 2018;19:672-81.

33. Eggermont AM, Chiarion-Sileni V, Grob JJ, et al. Prolonged survival in stage III melanoma with ipilimumab adjuvant therapy. N Engl J Med 2016;375:1845-55.

34. WeberJ, Mandala M, Del Vecchio M, et al. Adjuvant nivolumab versus ipilimumab in resected stage III or IV melanoma. NEngl J Med 2017;377:1824-35.

35. Eggermont AMM, BlankCU, Mandala M, et al. Adjuvant pembrolizumab versus placebo in resected stage III melanoma. N Engl J Med 2018;378;1789-801.

36. Rozeman EA, Menzies AM, van Akkooi ACJ, et al. Identification of the optimal combination dosing schedule of neoadjuvant ipilimumab plus nivolumab in macroscopic stage III melanoma (OpACIN-neo): a multicentre, phase 2, randomised, controlled trial. Lancet Oncol 2019;20:948-60.

37. Weber JS, Tang H, Hippeli L, et al. Serum IL-6 and CRP are prognostic indicators in melanoma patients receiving single agent and combination checkpoint inhibition [abstract 100]. J Clin Oncol 2019;37:. [Available online at: https://ascopubs.org/doi/ abs/10.1200/JCO.2019.37.15_suppl.100; cited 30October 2019]

38. Korn EL, Liu PY, Lee SJ, et al. Meta-analysis of phase II cooperative group trials in metastatic stage IV melanoma to determine progression-free and overall survival benchmarks for future phase II trials. J Clin Oncol 2008;26:527-34.

39. Ascierto PA, Melero I, Bhatia S, et al. Initial efficacy of antilymphocyte activation gene-3 (anti-LAG-3; BMS-986016) in combination with nivolumab (nivo) in pts with melanoma (MEL) previously treated with anti-PD-1/PD-L1 therapy [abstract 9520].JClin Oncol 2017;35:. [Available online at: https:// ascopubs.org/doi/abs/10.1200/JCO.2017.35.15_suppl.9520; cited 30 October 2019] 